\title{
Whole Exome Sequencing Revealed a Novel Sequence Variant in The OTULIN Underlying Auto- Inflammatory Syndrome
}

\section{Rubab Raza}

Quaid-i-Azam University

Raul Jimenez-Heredia

Medical University of Vienna

Muhammad Zeeshan Anwar

National University of Medical Sciences

Asmat Ullah

Quaid-i-Azam University

Ayisha Zia

Quaid-i-Azam University

\section{Sajid Rashid}

Quaid-i-Azam University

\section{Wasim Ahmad}

Quaid-i-Azam University

\section{Ana Krolo}

Medical University of Vienna

\section{Kaan Boztug}

Medical University of Vienna

Iffan Raza ( $\nabla$ siraza.pk@gmail.com )

HBSMDC: HBS Medical and Dental College https://orcid.org/0000-0002-7998-7404

\section{Research Article}

Keywords: OTULIN, Next Generation Sequencing, Flow cytometry, Mutation, Auto-inflammation

Posted Date: February 15th, 2021

DOI: https://doi.org/10.21203/rs.3.rs-164929/v1

License: (1) (1) This work is licensed under a Creative Commons Attribution 4.0 International License.

Read Full License 


\section{Abstract}

Purpose Systemic auto-inflammatory diseases are a diverse group of heterogeneous disorders resulting in development of the systemic inflammation in absence of the inflammatory induction. Sequence variants in the OTULIN gene, which disrupts its ubiquitination activity lead to auto-inflammation, panniculitis, and dermatosis syndrome. To date, only few disease-causing variants in the OTULIN have been reported.

In the study, presented here, sequence analysis of the OTULIN gene in a patient, exhibiting features of OTULIN-related auto-inflammatory syndrome (ORAS), revealed a novel disease-causing missense variant p.(Thr312Met). Further, effect of the variant on structure and function of the OTULIN protein has been examined using in silico OTULIN ${ }^{W T}$ and OTULIN ${ }^{\top 312 M}$.

Methods Cells, collected from the patient blood, were examined by flow cytometry. Search for the diseasecausing variants was carried out using exome followed by Sanger sequencing. Effect of the sequence variant on structure of the mutated protein was studied using in-silico analyses.

Results Flow cytometry analysis revealed slightly reduced number of lymphocytes, marked leukocytosis, and mildly increased levels of IgG. Whole exome sequencing coupled with Sanger sequencing revealed a homozygous missense variant [c.935C >T; p.(Thr312Met)] in the OTULIN gene. In-silico analyses revealed that the missense variant reduces OTULIN's expression and promotes accumulation of LUBAC-linked UB chains leading to auto-inflammation.

Conclusion Taken together, OTULIN may act as a novel therapeutic target for the development of immunomodulatory drugs that may potentially increase or stabilize their expression. Targeting more components of the Ub-proteasome pathway may provide new opportunities for therapeutic exploitation and drug discovery.

\section{Introduction}

Systemic auto-inflammatory diseases (SAIDs) are heterogeneous group of disorders characterized by spontaneous development of systemic inflammation leading to a variety of organ-specific manifestations. Cytokines are key regulators of the immune system which alert and activate lymphocytes. In time activation of the innate immune response is indispensable for proper tissue homeostasis and host defense. Disruption in regulation of these responses may lead to autoinflammatory diseases which are often considered as life-threatening [1].

Regulation of signaling cascades such as interleukin-1 (IL-1), type I interferon, or tumor necrosis factor (TNF) signaling, or activation of nuclear factor-KB (NF-KB) transcription factors rely upon protein ubiquitination - a versatile post translation modification $[1,2]$. Ubiquitin (Ub) mediates most of its cellular functions through structurally and functionally distinct polyUb signals [2,3]. These polyUb chains are linked either by one of seven Ub Lys residue or Ub Met1 (M1) generating M1 chains. The M1-linked chains 
dependent activation and regulation of NF-KB transcription factors is essential to control inflammation and immunity $[4,5,6]$. According to Hrdinka \& Gyrd- Hansen, ubiquitin chains depend on cytokine receptors. These receptors, upon stimulation, trigger the assembly of multi-protein receptor signaling complexes (RSCs) which include LUBAC, present in association with E3 Ub ligases [7]. Chains of linearly arranged ubiquitin molecules through $\mathrm{M} 1$ are generated by the linear ubiquitin assembly complex (LUBAC) comprising of HOIP, HOIL-1, and SHARPIN.

The LUBAC activity is regulated by OTULIN (MIM 617099), which is a highly conserved deubiquitinating enzyme with a specific activity to hydrolyze linear M1-linked Ub chains $[8,9,10]$. The gene OTULIN, also known as FAM105B or Gumby, localizes on chromosome 5p15.2 [11]. There are several negative regulators of NF-kB signaling such as A20, CYLD, and Cezanne which are essential for managing inflammation and the return to homeostasis [12]. Until now, only few mutations have been reported in OTULIN, causing OTULIN-related auto-inflammatory syndrome (ORAS). In the present study whole exome sequencing coupled with Sanger sequencing revealed a novel homozygous missense mutation in the OTULIN gene in a consanguineous Pakistani family causing ORAS. Further effects of the mutation have been studied using in silico OTULIN ${ }^{\text {WT }}$ and OTULIN ${ }^{\text {T312M }}$.

\section{Methods}

\section{Study Oversight}

The studies including immunologic diagnostic procedures and genetic analyses were performed in accordance with guidelines of good clinical practice, the current version of the Declaration of Helsinki, with written informed consent from the patients or patient's legal representatives, and with approval from the relevant Institutional Review Boards.

\section{Material Sampling}

Blood samples were collected from the patient and available parent. DNA was extracted using the GenElute $^{T M}$ blood genomic DNA kit (Sigma-Aldrich, St. Louis, MO, USA). DNA quantification was performed using Nanodrop1000 spectrophotometer (Thermal scientific, Wilmington, MA, USA) to calculate the optimal density at $260 \mathrm{~nm}$.

\section{Whole Exome Sequencing and Analysis}

Whole exome sequencing was performed using a TruSeq Rapid Exome kit as well as the lllumina HiSeq3000/4000 system and the cBot cluster generation instruments as previously described with minor changes $[13,14]$. Briefly, reads were aligned to the human genome version 19 by means of the BurrowsWheeler Aligner (BWA). VEP was used for annotating single nucleotide variants (SNVs) and insertions/deletions lists. The obtained list was then filtered according to the presence of variants with a minor allele frequency (MAF) >0.01 in 1,000 Genomes, gnomAD, and dbSNP build 149. After further filtering steps for nonsense, missense, and splice-site variants using VCF. Filter software, an internal 
database was used to filter for recurrent variants [15]. Moreover, variants were prioritized using tools, such as SIFT, Polyphen-2 and the combined annotation dependent depletion (CADD) score, that predict the deleteriousness of a present variant $[16,17]$.

\section{Sanger Sequencing}

Sanger sequencing was used to validate the variant found in the OTULIN. This was carried out by designing the following set of primers for PCR-amplification of the variant.

Fw: 5'-AAAAACGTTGTGGCTTCACC-3'

Rv: 5'-ATAGTGCCGATCGTCCTCAG-3'

\section{Data Set}

The crystal structure of human OTULIN (PDB ID: 3ZNV) was retrieved through protein data bank (PDB) [18]. The energy minimization steps were carried out by UCSF Chimera 1.5.6 using conjugate gradient algorithm and Amber force field. 3D structure of OTULIN ${ }^{\top 312 M}$ was predicted by Modeller 9.14 using OTULIN ${ }^{W T}$ structure as template $[19,20]$. The predicted 3D structure was validated by MolProbity analysis, followed by structure optimization through WinCoot $[21,22]$.

\section{Molecular Dynamics Simulations}

OTULIN ${ }^{\mathrm{WT}}$ and OTULIN ${ }^{\mathrm{T} 312 \mathrm{M}}$ systems were simulated through Amber16 [23]. In order to the check parameters including bonds, angles, or dihedrals, parmchk utility was used to generate frcmod file to load in LEaP for adding missing parameters. All hydrogen atoms were added using the Leap module in the AMBER package. Topology and rst7 files were generated using tleap program through leaprc.protein.ff14SB force field [24]. For the required hydration of all systems, we used TIP4P water box with a buffer distance of at least $10 \AA$ around the solute [25]. The initial minimization was done holding the restraints over the solute for 500 steps using the steepest descent algorithm followed by another 500 steps with a conjugate gradient process. Another round of minimization was carried out without any restraints for 3000 steps. Both minimizations were carried out using the cut off value of $12 \AA$. Heating dynamics was applied to both complexes at $300 \mathrm{~K}$. Langev in dynamics was used for temperature maintenance. Whole arrangement was optimized, thermalized, and equilibrated using standard equilibration protocol. All simulations were carried out using periodic boundary conditions under isothermal and isobaric conditions $(T=300 \mathrm{~K} ; \mathrm{p}=1 \mathrm{~atm})$. The Berendsen thermostat was used for controlling the temperature. The shake algorithm was used to restrain all the bonds at the time step of 2 fs [26]. Finally, MD simulation run was performed for $100 \mathrm{~ns}$. After each MD run, trajectory files were generated and analyzed using cpptraj [27]. Comparative analysis of Root mean square deviation (RMSD) and Root mean square fluctuations (RMSF) were performed for both systems. For the visualization of the results, UCSF Chimera 1.5.6 was deployed. 


\section{Results}

\section{Clinical Evaluation}

A male child of Pakistani origin was born to consanguineous parents at 36 weeks gestation via spontaneous vaginal delivery at Military Hospital Rawalpindi, Pakistan. He is the first child with unremarkable family history. It was a booked pregnancy with normal antenatal course. He had a low birth weight of 4 pounds but good APGAR (Appearance, Pulse, Grimace, Activity, and Respiration) score of $8 / 10$ and no dysmorphism. He remained admitted in nursery for low birth weight and respiratory distress. He was managed conservatively and discharged on 8th day after circumcision. At $3^{\text {rd }}$ day of birth, he developed right upper arm abscess at BCG vaccination site that required IV antibiotics treatment. The child developed abscess at the circumcision site and was treated with local antibiotic dressing. Umbilical cord detached on 10th day with normal healing. At one month of age he developed abscesses at wrist and elbow and was treated with IV antibiotics. At 6 months of age he developed cellulitis of right thigh following intramuscular vaccination and required surgical debridement and IV antibiotics. During first year of life he had recurrent diarrhea, skin abscesses and three episodes of pneumonia that required hospital admissions, however, no organism could be isolated. He had poor weight gain but attained normal developmental milestones. He had no visceromegaly, lymphadenopathy or rash. Due to recurrent skin abscesses and pneumonia, the child died at the age of 19 months (Fig. 1a).

\section{Clinical Laboratory Tests}

Complete blood count was performed on two occasions. He suffered with neutrophilic leukocytosis. The leukocyte counts were $22000 /$ ul. Neutrophils were $81 \%$ mostly with toxic granulation. Lymphocyte subset analysis at 1 year showed markedly depressed lymphocytes (6\%), low CD3 T cells (31\%), CD4 and CD8 T lymphocytes $22 \%$ and $8 \%$, respectively, and a slightly raised CD4:CD8 ratio (2.75). However, absolute lymphocyte count was not low due to marked leukocytosis $(61200 / \mathrm{LL})$. CD19 positive B cells and NK cells were in normal range. At the age of one year, IgA and IgM levels were $40 \mathrm{mg} / \mathrm{dl}$ and $42 \mathrm{mg} / \mathrm{dl}$, respectively. However, IgG level was slightly raised to $902 \mathrm{mg} / \mathrm{dl}$ (Table 1).

\section{Genetic Investigation}

The detailed family history and pedigree analysis of the family suggested that the patient suffered from a disorder segregating in an autosomal recessive manner. Using DNA of the patient, whole-exome sequencing (WES) was performed as described before revealing 17 different homozygous variants (Supplementary Table S1) [28]. Sanger sequencing of the homozygous C-to-T substitution in chr5:14693033 [ENST00000284274.4:c.935C>T, p.(Thr312Met)] confirmed partial segregation (due to absence of one parent) of the OTULIN gene within the family (Fig. 1b). This missense change replaced an essential polar amino acid threonine with sulphur containing non-polar methionine. The OTULIN ${ }^{T 312 M}$ missense mutation lies in the C-terminal ovarian tumor (OTU) domain (79-352 aa) that mediates deubiquitinase activity of the protein (Fig. 1c). The OTULIN ${ }^{\top 312 M}$ is only the 4 th missense mutation reported in the gene. Previously two missense mutations p.(Leu272Pro), p.(Tyr244Cys) and one 
frameshift mutation p.Gly174Aspfs*2 was reported by Zhou et al. in 2016 and another missense mutation p.(Gly281Arg) was reported by Damgaard et al. in $2019[29,30]$. The mutation reported in this manuscript (p.Thr312Met) is not present in the GnomAD and 1,000 Genome database. Moreover, SIFT and Polyphen scores confirmed the deleterious nature of this mutation. The mutation carried a combined annotation-dependent depletion (CADD) score of 27 (Supplementary Table S1).

\section{Mutation of T312M Reduces OTULIN}

OTULIN is a 352-residue protein that consists of an N-terminal LUBAC-binding PUB-interacting motif (PIM), a C-terminal ovarian tumor (OTU) domain that mediates deubiquitinase activity of OTULIN (79-352 aa) and a putative C-terminal class I PDZ-binding motif (PDZbm: ETSL) (Fig. 1c). In the modelled OTULIN ${ }^{\top 312 M}$, Ramachandran plot indicated the presence of $>95 \%$ residues in the sterically allowed region (Fig. 1d and e) (Supplementary Table S2). Moreover, parameters like peptide bond planarity; nonbonded interactions, Ca tetrahedral distortion, main chain H-bond energy and G-factor for the OTULIN ${ }^{\top 312 M}$ structure were observed in the favorable ranges. Through superimposition of I domains of OTULIN ${ }^{T 312 M}$ and OTULIN ${ }^{W T}$ structures, an overall RMSD value of $1.12 \AA$ was observed (Supplementary Figure S1).

\section{Molecular Dynamics (MD) Analysis}

In order to monitor the detailed time-dependent conformational changes, 100 ns MD simulation runs were performed using OTULIN ${ }^{W T}$ and OTULIN ${ }^{\top 312 M}$ structures. Resulting trajectories were carefully analyzed to determine the stability and structural properties during MD simulations. To evaluate the stability and fluctuations of a-carbon atoms, time series plots of root mean square deviation (RMSD) and root mean square fluctuation (RMSF) were generated. For OTULIN ${ }^{W T}$, RMSD plot demonstrated system stability in the range of $2.3 \AA$, while it was slightly higher $(2.7 \AA)$ in case of OTULIN ${ }^{\top 312 M}$ (Fig. 2a). RMSF analysis indicated that the residues ranging from T1118-V123 and P146-Q170 exhibited more fluctuations in OTULIN $^{\text {T312M }}$ (Fig. 2b).

In both OUTLIN ${ }^{W T}$ and OTULIN ${ }^{\top 312 M}$, the overall secondary structure topology was similar with minor changes. A-helices were altered due to positional differences of individual residues, length variation and helical breaks. Overall, two $\beta$ strands (S119-V123 and Y340-V344) were converted into loops in OTULIN ${ }^{T 312 M}$. P147-Q150 and L157-Q170 helical regions were elongated into single helix (P146-Q170) in OTULIN $^{\text {T312M }}$. Another helix (Q288-V298) was modified into M291-R299, while R263-S266 helix was converted into loop in OTULIN ${ }^{\top 312 M}$. Similarly, 188-G95 helix was reduced in OTULIN ${ }^{\top 312 M}$ by two residues (188-K93) (Fig. 2c and d). The 2-dimension representation is shown in Supplementary Figure S2.

\section{Discussion}

Protein ubiquitination is a key posttranslational modification that regulates almost every aspect of cellular homeostasis through structurally and functionally distinct polyubiquitin signals [2]. Ubiquitin 
molecules are linked either via one of $7 \mathrm{Ub}$ Lys $(\mathrm{K})$ residues or via Ub M1, forming M1-linked chains. The linkage between $\mathrm{Ub}$ residues is generated by the linear ubiquitin chain assembly complex (LUBAC) that is comprised of HOIP, HOIL-1, and SHARPIN. The M1-linked Ub chains regulate the immune responses through regulation of NF-KB immune homeostasis and responses to infection $[4,31,32]$. OTULIN is the only deubiquitinase (DUB) that specifically cleaves M1 linkages $[9,10]$. Previously it has been established that OTULIN directly binds with HOIP component of LUBAC, and OTULIN-knockdown cell line study demonstrates increased M1-linked chains on LUBAC and its substrates, which confirms its pivotal role as a deubiquitinase $[9,10,33,34,35]$.

In the present study, whole exome sequencing identified a novel missense mutation p.(Thr312Met) in the OTULIN gene. This mutation is localized in the OTU domain, which contains deubiquitinase activity. OTULIN ${ }^{T 312 M}$ leads to a spectrum of clinical manifestations such as persistent bowel symptoms, pus from skin abscess, diarrhea, pneumonia, and fever. All these clinical features are coherent to those reported earlier by Fiil \& Gyrd-Hansen (2016) and Damgaard et al. (2019) OTULIN L272P and OTULIN G281R, respectively $[30,36]$. However, such patients do not have any clear finding pointing to primary immunodeficiency in routine immunological workup. Serum immunoglobulin levels could be low, raised, or normal, so are lymphocyte subclasses. Although we did not check antibody responses to vaccination since vaccination history was not available, such responses are expected to be normal in such patients and hardly provide any help in making diagnosis [29].

Based on the functional studies, OTULIN ${ }^{\top 312 \mathrm{M}}$ leads to accumulation of LUBAC-linked UB chains, further associated with enhanced NF-KB activity and increased TNFR1 signaling $[9,10,33,34,35]$. This would result in a systemic auto-inflamatory disease affecting different organs. The phenotype could be lethal if left untreated. In the present study, marked lymphocytopenia and leukocytosis with increased circulating neutrophils were observed in accordance with the recent reports $[30,36]$. Remarkably, OTULIN ${ }^{\top 312 M}$ did not affect CD19 and NK cells along with immunoglobulin levels, which were found in healthy range.

In-silico analysis revealed that OTULIN ${ }^{\top 312 M}$ may lead to functional disturbances. The comparative structural analysis of OTULIN ${ }^{W T}$ and OTULIN ${ }^{\top 312 M}$ revealed the presence of multiple conformational transitions at the secondary structure level that may arise due to shifting of a polar to a nonpolar residue. The noticeable differences were the absence of two $\beta$-strands: S119-V123 and Y340-V344 and merging of two helices (P147-Q150 and L157-Q170) into single helix (P146-Q170) in OTULIN ${ }^{T 312 M}$. Recent mutational spectrum analysis for the catalytically inactive OTULIN ${ }^{\mathrm{C} 129 \mathrm{~A}}$ that leads to autoubiquitination of HOIP via Met1-linked polyUb chains suggests the importance of OTU domain in maintaining the stability of functional OTULIN-HOIP complex $[9,35]$. Thus, any alteration in the conformation of interface required for the assembly of M1-polyUb to OTU domain may hinder the LUBAC-dependent signaling, required for cellular homeostasis (Fig. 3).

\section{Conclusion}


Taken together, we describe in this study a new mutation affecting the OTULIN-HOIP complex. With the information extracted from functional and computational analysis, we can conclude that OTULIN may act as a novel therapeutic target for the development of immunomodulatory drugs that may potentially increase or stabilize their expression. One of the major challenges for finding effective drugs would be in developing cell-based therapies. The ubiquitination is a very versatile process which regulates almost every step of cellular homoeostasis and is regulated at multiple levels [2]. Targeting more components of the Ub-proteasome pathway may provide new opportunities for therapeutic exploitation and drug discovery.

\section{Declarations}

Funding Not applicable

Conflict of Interest: The authors declare that they have no conflict of interest.

Ethical Approval: Study is approved from relevant Institutional Review Boards.

Consent to Participate Written informed consent from the patients or patient's legal representatives were taken

Code Availability Not applicable

Authors' Contributions All authors contributed to the design and conception of the study.

Sample collection and analysis were performed by [Rubab Raza], [Muhammad Zeeshan Anwar] and [Syed Irfan Raza].

Clinical details: [Muhammad Zeeshan Anwar], ; Manuscript write up: [Rubab Raza, Syed Irfan Raza, Raul Jimenez-Heredia]; Research coordinator and Data analysis: [Syed Irfan Raza]; Sanger Sequencing: [Asmat Ullah]; Insilico analysis: [Ayisha Zia, Sajid Rahsid]; Study Supervisor (Pakistan): [Wasim Ahmad]; Next Generation Sequencing and genetic data analyses: [Raul Jimenez-Heredia, Ana Krolo]; research supervisor (Vienna): [Kaan Boztug]. All authors reviewed and approved the final manuscript.

\section{References}

1. Nathan C, Ding A. Nonresolving inflammation. Cell. 2010;140:871-882.

2. Komander D, Rape M. The ubiquitin code. Annu Rev Biochem. 2012;81:203-229.

3. Swatek KN, Komander D. Ubiquitin modifications. Cell Res. 2016;26:399-422.

4. Bonizzi G, Karin M. The two NF-kappaB activation pathways and their role in innate and adaptive immunity. Trends Immunol. 2004;25:280-288.

5. Jiang $X$, Chen ZJ. The role of ubiquitylation in immune defence and pathogen evasion. Nat Rev Immunol. 2012;12:35-48. 
6. Etzioni A, Ciechanover A, Pikarsky E. Immune defects caused by mutations in the ubiquitin system. J Allergy Clin Immunol. 2017;139:743-753.

7. Hrdinka M, Gyrd-Hansen M. The Met1-Linked Ubiquitin Machinery: Emerging Themes of Deregulation. Mol Cell.2017;68(2):265-

8. Walczak H, Iwai K, Dikic I. Generation, and physiological roles of linear ubiquitin chains. BMC Biol. 2012;10:23.

9. Keusekotten K, Elliott PR, Glockner L, Fiil B, Damgaard RB, Kulathu Y, Wauer T, Hospenthal MK, GyrdHansen $M$, Krappmann $D$ et al. OTULIN antagonizes LUBAC signaling by specifically hydrolyzing Met1-linked polyubiquitin. Cell. 2013;153:1312-1326.

10. Rivkin E, Almeida SM, Ceccarelli DF, Juang Y-C, MacLean TA, Srikumar T, Huang H, Dunham WH, Fukumura $\mathrm{R}$, Xie $\mathrm{G}$ et al. The linear ubiquitin-specific deubiquitinase gumby regulates angiogenesis. Nature. 2013;498:318-324.

11. Gross MB. Personal Communication. Baltimore, Md. 3/25/2014.

12. Harhaj EW, Dixit VM. Regulation of NF-KB by deubiquitinases. Immunol Rev. 2012;246(1):107-

13. Salzer E, Cagdas D, Hons M, Mace EM, Garncarz W, Petronczki OY et al. RASGRP1 deficiency causes immunodeficiency with impaired cytoskeletal dynamics. Nat Immunol. 2016;17(12):1352-1360.

14. Ozen A, Comrie WA, Ardy RC, Dominquez CC, Dalgic B, Beser OF et al. CD55 Deficiency, Early-Onset Protein-Losing Enteropathy, and Thrombosis. N Engl J Med. 2017;377(1):52-61.

15. Müller H, Jimenez-Heredia R, Krolo A, Hirschmugl T, Dmytrus J, Boztug K, Bock C. VCF.Filter: Interactive prioritization of disease-linked genetic variants from sequencing data. Nucleic Acids Res. 2017;45(W1):W567-W572.

16. Adzhubei IA, Schmidt S, Peshkin L, Ramensky VE, Gerasimova A, Bork P, Kondrashov AS, Sunyaev SR. A method and server for predicting damaging missense mutations. Nat 2010;7(4):248-9.

17. Kircher M, Witten DM, Jain P, O'Roak BJ, Cooper GM, Shendure J. A general framework for estimating the relative pathogenicity of human genetic variants. Nat Genet. 2014;46:310.

18. Thanki N, Weissig H, Westbrook J. The protein data bank. Section D: Biological .... 2002.

19. Pettersen EF, Goddard TD, Huang CC, Couch GS, Greenblatt DM, Meng EC, et al. UCSF Chimera-a visualization system for exploratory research and analysis. J Comput Chem. 2004;25(13):16051612.

20. Potterton L, Yuan F, van Vlijmen H, M Karplus. Evaluation of comparative protein modeling by MODELLER. 1995;23(3):318-26.

21. Chen VB, Arendall WB, Headd JJ, DA Keedy, Immormino RM Kapral GJ, Murray LW, Richardson JS, Richardson DC. MolProbity: all-atom structure validation for macromolecular crystallography. Acta Crystallogr D Biol Crystallogr. 2010;66(Pt 1):12-

22. Emsley P, Lohkamp B, Scott WG, Cowtan K. Features, and development of Coot. Acta Cryst.2010;D66:486-501.

23. DA Case, Cerutti DS, Cheatham T, Darden T, Duke R. AMBER16. San Fr Univ Calif. 2016. 
24. Maier JA, Martinez C, Kasavajhala K, Wickstrom L, Hauser KE, Simmerling C. ff14SB: Improving the Accuracy of Protein Side Chain and Backbone Parameters from ff99SB. J Chem Theory Comput. 2015;11(8):3696-3713.

25. Jorgensen WL, Chandrasekhar J, Madura JD. Comparison of simple potential functions for simulating liquid water. J Chem Phys. 1983;79:926.

26. Berendsen HJC, Postma JPM. Molecular dynamics with coupling to an external bath. J Chem Phys. 1983;81:3684.

27. Roe DR, Cheatham TE. PTRAJ and CPPTRAJ: software for processing and analysis of molecular dynamics trajectory data. J Chem Theory Comput. 2013;9(7):3084-3095.

28. Thian M, Hoeger B, Kamnev A, Poyer F, Köstel Bal S, Caldera M, Jiménez-Heredia R, Huemer J, Pickl WF, Groß M, Ehl S, Lucas CL, Menche J, Hutter C, Attarbaschi A, Dupré L, Boztug K. Germline biallelic PIK3CG mutations in a multifaceted immunodeficiency with immune dysregulation. Haematol. 2020;105(10):231399

29. Zhou Q, Yu X, Demirkaya E, Deuitch N, et al. Biallelic hypomorphic mutations in a linear deubiquitinase define otulipenia, an early onset autoinflammatory disease. Proc Natl Acad Sci. 2016;113(36):10127-

30. Damgaard RB, Elliot PR, Swatek KN, Maher ER, Stepensky P, Elpeleg O, Komander D, Berkun Y. OTULIN deficiency in ORAS causes cell type specific LUBAC degradation, dysregulated TNF signalling and cell death. EMBO Mol Med. 2019;11(3):e9324

31. Fiil BK, Gyrd-Hansen M. Met1-linked ubiquitination in immune signaling. FEBS J. 2014;281(19):4337-

32. Iwai K, Fujita H, Sasaki Y. Linear ubiquitin chains: NF-KB signalling, cell death and beyond. Nat Rev Mol Cell Biol. 2014;15(8):503-

33. Elliot PR, Mielsen SV, Marco-Casanova P, Fiil BK, et al. Molecular basis and regulation of OTULINLUBAC interaction. Mol Cell. 2014;54(3):335-

34. Schaeffer V, Akutsu M, Olma MH, Gomes LC, et al. Binding of OTULIN to the PUB domain of HOIP controls NF-KB signaling. Mol Cell. 2014;54(3):349-

35. Fiil BK, Damgaard RB, Wagner SA, Keusekotten K, et al. OTULIN restricts Met1-linked ubiquitination to control innate immune signaling. Mol Cell. 2013;50(6):818-

36. Fiil BK, Gyrd-Hansen M. OTULIN deficiency causes auto-inflammatory syndrome. Cell Res. 2016;26(11):1176-1177.

\section{Table}

Table 1 Lymphocytes subsets and reference values at childhood 


\begin{tabular}{|llll|}
\hline S No. & Parameter & Count $(\%)$ & Ref range count (\%) \\
\hline 1 & TLC & $61200 / \mathrm{ul}$ & $6000-17500$ \\
3 & Lymphocyte & $3672(6)$ & $3320-7006$ \\
4 & CD3+ T cells & $1138(31)$ & $2284-4776(51.8-74.2)$ \\
5 & CD3+4+ T cells & $808(22)$ & $1523-3472(34.9-53.1)$ \\
6 & CD3+8+ T cells & $294(8)$ & $524-1583(12.8-27.1)$ \\
7 & CD16+56+ NK cells & $551(15)$ & $230-801(4.0-15.1)$ \\
\hline Patient Immunoglobulin levels & & \\
\hline 8 & IgG & $902 \mathrm{mg} / \mathrm{dl}$ & $11-769 \mathrm{mg} / \mathrm{dl}$ \\
9 & IgA & $40 \mathrm{mg} / \mathrm{dl}$ & $11-221 \mathrm{mg} / \mathrm{dl}$ \\
10 & IgM & $42 \mathrm{mg} / \mathrm{dl}$ & $4-110 \mathrm{mg} / \mathrm{dl}$ \\
\hline
\end{tabular}

Figures 

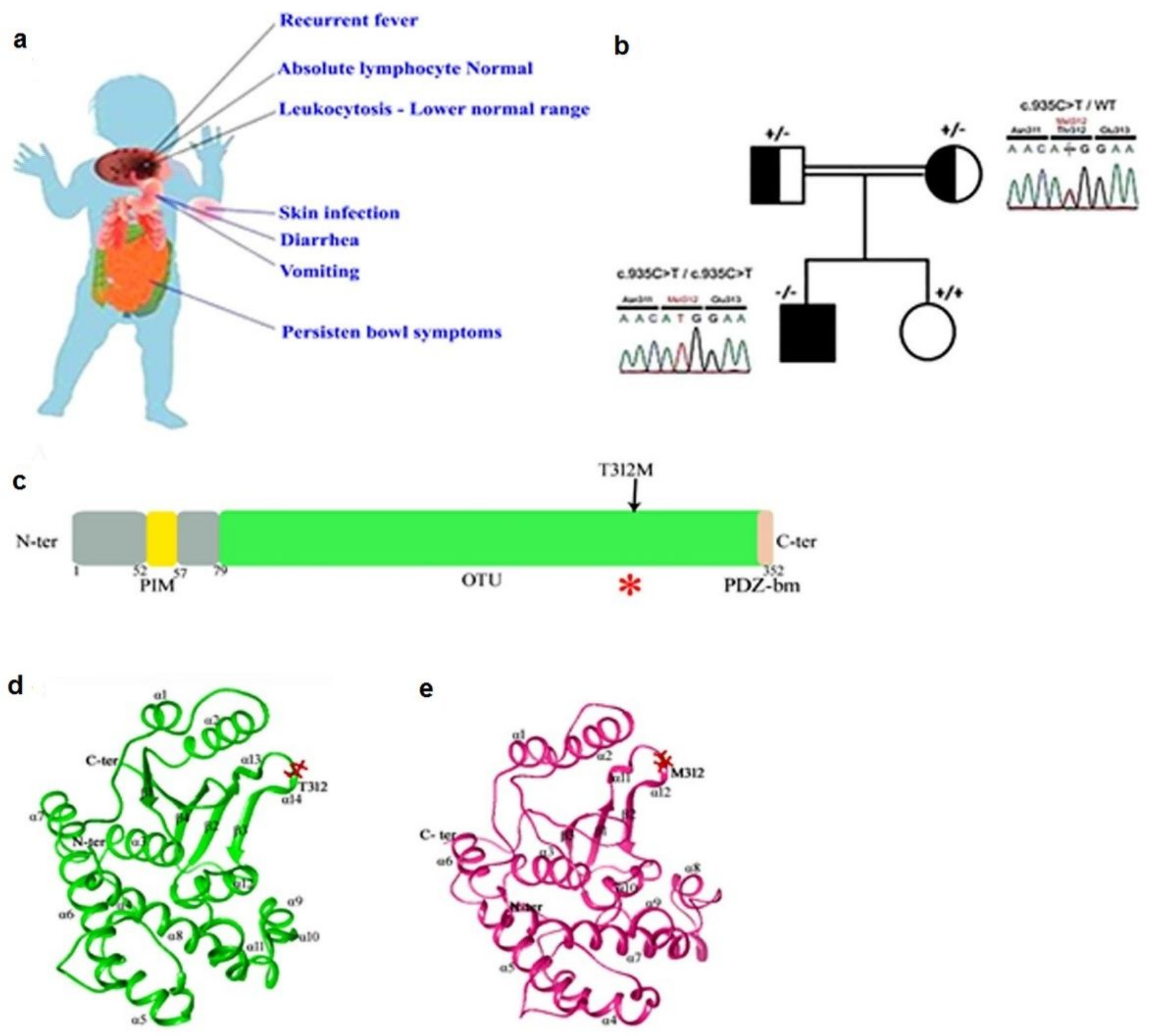

Figure 1

a) Sketch showing the different clinical manifestations in the patient. b) Familial segregation of the identified OTULIN (ENST00000284274.4:c.935C >T, p. Thr312Met) mutation in the family, indicating an autosomal-recessive pattern of inheritance. Circles are showing females while squares are representing males. A double line between parents indicates consanguinity. c) Domain-wise representation of OTULIN consisting of an N-terminal LUBAC-binding PUB-interacting motif (PIM) (yellow), a C-terminal ovarian tumor (I) domain (green) and class I PDZ-binding motif (PDBZ-bm) (pink). T312M point mutation is located in the (OTU) domain, indicated by black arrow. d) The crystal structure of OTULINWT (PDB ID: 
3ZNZ). e) Modelled OTULINT312M structure. T312 and M312 residues are indicated in red colored ball and stick representation and labeled in black.
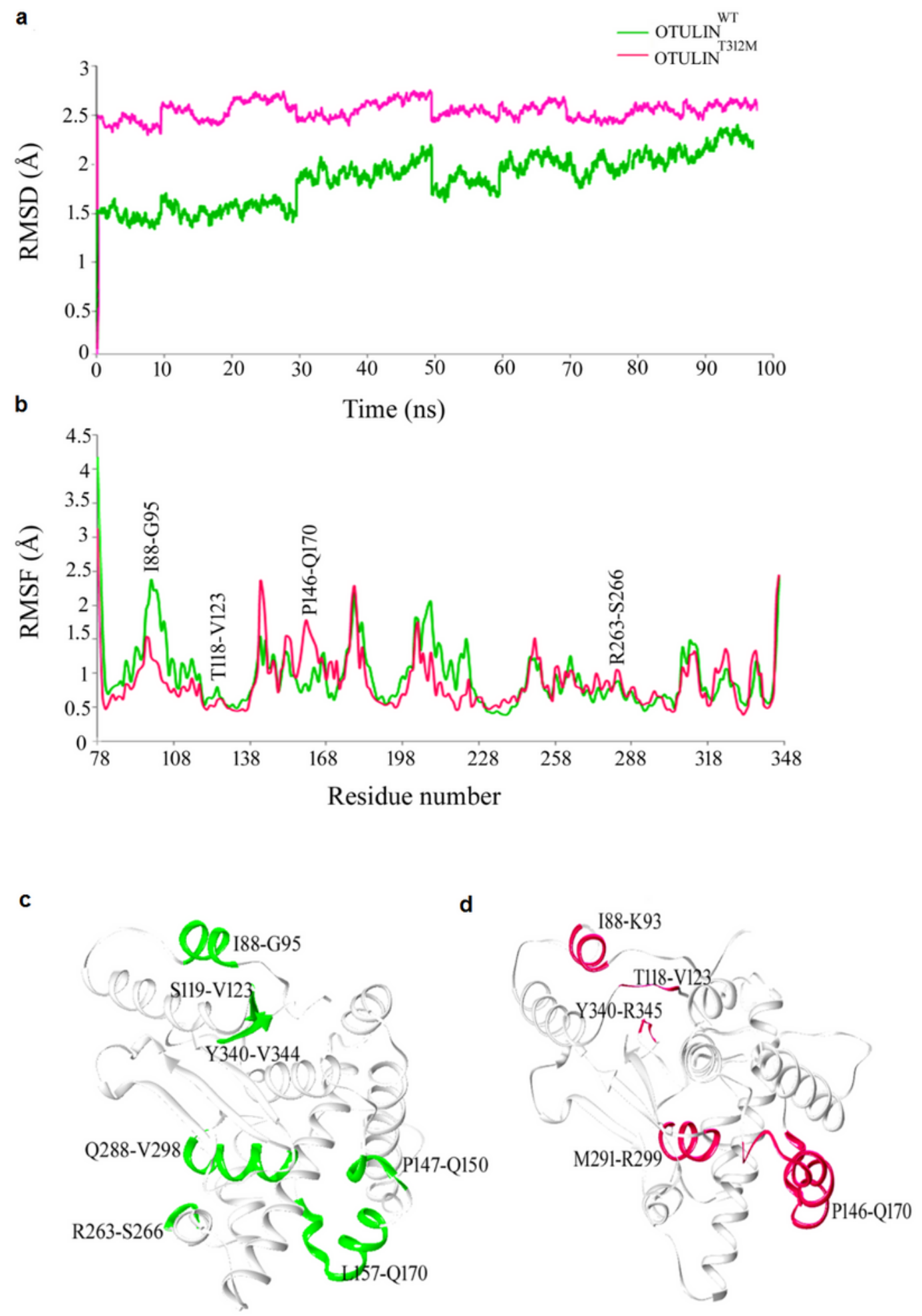

Figure 2

MD simulation analysis. a) RMSD plot and b) RMSF plot for OTULINWT (green) and OTULINT312M (pink), demonstrating the fluctuating residues (in black). Structural differences are indicated in green and pink colors in c) OTULINWT and d) OTULINT312M, respectively. 

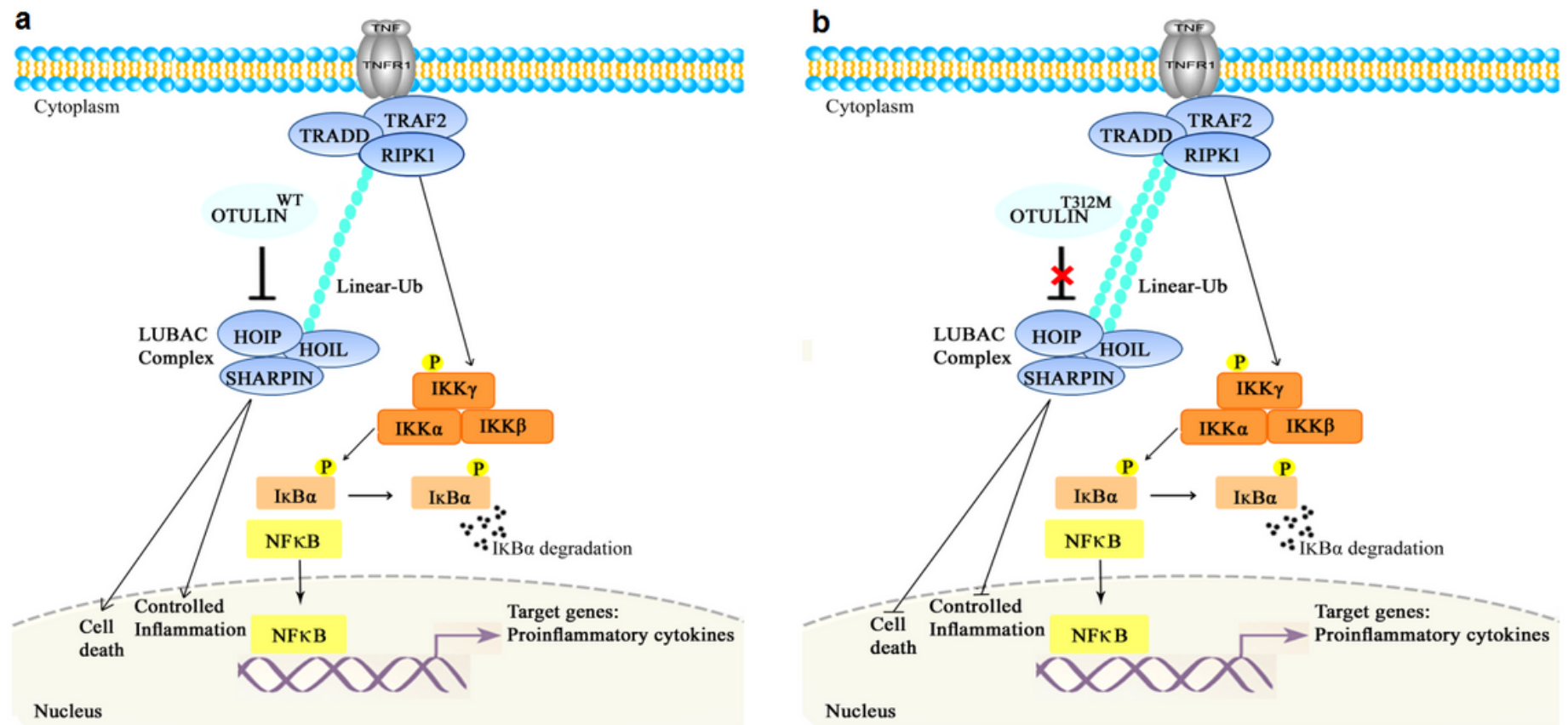

\section{Figure 3}

Schematic representation of OTULIN-dependent signaling to elaborate the catalytic mechanism of a) OTULINWT and b) OTULINT312M. The canonical NF-KB pathway is regulated by linear M1-linked Ub chains. RIPK1 (Receptor-interacting serine/threonine-protein kinase 1) is the central adaptor that is required for the assembly of TNFR1 (Tumor necrosis factor receptor 1) receptor signaling complex. Polyubiquitylated RIPK1 mediates recruitment of IKK (Inhibitor of nuclear factor-KB (IKB) kinase) complex that is another target for ubiquitination. The activated IKK complex phosphorylates an inhibitor of kappa $\mathrm{B}(\mathrm{I} \mathrm{KBa})$ and targets $\mathrm{IKBa}$ for proteasome-mediated degradation. Linear Ub chains are added to RIPK1 and IKKY by LUBAC. OTULIN negatively regulates the NF-KB pathway by cleaving linear Ub chains from target molecules, RIPK1 and IKKY. Decreased expression of OTULIN in patients with ORAS (OTULINrelated auto-inflammatory syndrome) may lead to activation of the NF-KB pathway, increased expression of proinflammatory transcripts in immune cells, and systemic inflammation.

\section{Supplementary Files}

This is a list of supplementary files associated with this preprint. Click to download.

- SupplementalData.pdf 